\title{
GPU-accelerated Tersoff potentials for massively parallel Molecular Dynamics simulations
}

\author{
Trung Dac Nguyen ${ }^{1}$ \\ National Center for Computational Sciences, Oak Ridge National Laboratory, TN 37831, USA \\ Institute of Mechanics, Vietnam Academy of Science and Technology, Hanoi, Vietnam
}

\begin{abstract}
The Tersoff potential is one of the empirical many-body potentials that have been widely used in simulation studies at atomic scales. Unlike pair-wise potentials, the Tersoff potential involves three-body terms, which requires much more arithmetic operations and data dependency. In this contribution, we have implemented the GPU-accelerated version of several variants of the Tersoff potential for LAMMPS, an open-source massively parallel Molecular Dynamics code. Compared to the existing MPI implementation in LAMMPS, the GPU implementation exhibits a better scalability and offers a speedup of $2.2 \mathrm{X}$ when run on 1000 compute nodes on the Titan supercomputer. On a single node, the speedup ranges from 2.0 to 8.0 times, depending on the number of atoms per GPU and hardware configurations. Among the most notable features of our GPU-accelerated version include its design for MPI/accelerator heterogeneous parallelism, its compatibility with other functionalities in LAMMPS, its ability to give deterministic results and to support both NVIDIA CUDA- and OpenCL-enabled accelerators. Our implementation is now part of the GPU package in LAMMPS and accessible for public use.
\end{abstract}

Keywords: Tersoff, LAMMPS, GPU acceleration, hybrid MPI/GPU, high-performance computing.

\section{Introduction}

Empirical force fields such as Stillinger-Weber [1], Tersoff [2] and other bond-order potentials play a crucial role in capturing the many-body effects in atomic and molecular systems and in chemical reactions that involve metals, covalent solids and carbon nanotubes. Compared to pairwise potentials, these many-body potentials are characterized by their higher degree of computational complexity associated with heavy data dependency and arithmetic intensity. As the current priority for development of such force fields has been to improve the accuracy and transferability of these potentials [3], few efforts have been devoted to boosting their performance, except for relying on increased CPU speeds and core counts.

The introduction of general-purpose graphics processing units (GPGPUs) and many-core processors to scientific computing over the past decade has opened a new horizon for accelerating molecular dynamics codes. The fine-grained parallelism offered by these accelerators demands new approaches to parallelize the computationally intensive tasks and/or to off-load all the computation and necessary data to the device. The former option is often pursued by legacy codes (e.g., NAMD [4], GROMACS [5] and LAMMPS [6]), while the latter by more recent codes (e.g., HOOMD-Blue [7]). Most of the force fields ported to the GPU to date are pairwise potentials as it is fairly straightforward to implement them in the single-instruction multiple-thread model provided by GPGPUs.

Efforts to parallelize many-body potentials have recently been made in the literature $[8,9,10,11,12,13]$. Optimized implementation of many-body potentials for crystalline structures were proposed by Hou and coworkers, where the neighbor list of individual atoms are presumably stable during a simulation [10, 11]. Recently, Minkin et al. [13] demonstrated that their OpenCL implementation offers significant speedup for the embedded atom model (EAM) and Tersoff potentials on NVIDIA GPUs compared to serial runs. However, specifics on the benchmarked systems

\footnotetext{
${ }^{1}$ Email: nguyentd@imech.ac.vn
} 
(e.g. density and temperature) were not given in their study and the benchmark system size was shown only up to 16000 atoms. Open-source software packages such as HOOMD-Blue and LAMMPS also support GPU acceleration for the EAM and Tersoff potential $[8,9]$. Those implementations were highly optimized for NVIDIA GPUs, but to our knowledge the performance of the accelerated version of the Tersoff potential has not been available. To date there are three options for using the Tersoff potential with accelerators in LAMMPS. The first option is to use the USER-CUDA package, which was written for NVIDIA CUDA-enabled GPUs; however, this package is now deprecated and no longer under maintenance. The second option is available in the Kokkos library, which is designed for generic accelerators including GPUs and many-core coprocessors. The third option is provided by the USER-INTEL package, which is optimized for Intel MIC platforms [14]. At the time of this writing, the first report to evaluate the performances of the Tersoff potential using a vectorization implementation for the USER-INTEL and Kokkos packages has just been released [15].

The GPU package in LAMMPS is designed for heterogeneous computing platforms, i.e., multi-core CPUs and accelerators $[16,17,18]$. It allows for performing non-bonded force computation and running the long-range electrostatic solver on the accelerators concurrently with other force computes such as bonds, angles, dihedrals and impropers. We note that complex simulations in practice often involve significant computations that occur each timestep or every few timesteps, which at least in LAMMPS, have not been (and may never be) implemented for the GPU. Examples include specialized boundary conditions, application of constraint forces, simulation box deformations, calculation of diagnostic properties and so on. With the GPU package, these computations still run efficiently on the CPU via conventional MPI-based parallelization.

Over the past five years, the number of force fields supported by the GPU package has been increasing steadily, including commonly used pairwise potentials [16], EAM [9] and Stillinger-Weber potential [18] and dissipative particle dynamics [19]. The Stillinger-Weber potential was the first three-body potential to be added to the GPU package by Brown and Masako [18], which exhibits significant performance gain for simulations of the single-site $\mathrm{mW}$ water model across thousands of compute nodes on the Titan supercomputer. In their work, the system is single-component, and all the model water particles interact via the Stillinger-Weber potential with a single parameter set.

In the present study, we aim at accelerating the calculation of the Tersoff potential and its variants for scientific applications involving multi-element systems, liquid and solid phases and combined force fields. Based on the current framework of the GPU package, our present implementation also allows for 1) concurrent execution of non-accelerated and accelerated parts, 2) compatibility with other existing functionalities in LAMMPS, 3) deterministic results and 4) the use of CUDA and OpenCL-enabled accelerators. We will discuss in detail our implementation and characterize its performance on different systems and hardware platforms. Our GPU-accelerated implementation of the Tersoff potentials have been available in the main LAMMPS repository since November 2015.

\section{Methods}

\subsection{LAMMPS and the GPU package}

The primary parallelization scheme in LAMMPS is spatial decomposition, which was described in detail in Ref. [6]. For a spatial decomposition, the simulation box is divided into non-overlapping sub-domains, one per MPI task or processor. The processor is responsible for updating the forces, positions and velocities of "owned" atoms within its sub-domain. This requires communication with processors owning nearby sub-domains to acquire "ghost" atoms" coordinates, which contribute to the forces on owned atoms. The thickness of the buffer region containing the "ghost" atoms for a given sub-domain is typically determined by the maximum cutoff distance of the non-bonded interactions, namely pair styles in LAMMPS, in use. The cutoff distance defined by the non-bonded force fields is used to construct the neighbor list of individual atoms, which is invariant during a certain number of time steps, under the assumption that the atoms would not move farther than half some skin distance over that time period. This assumption can be justified at the end of the run by the number of dangerous builds being zero.

The GPU package was first introduced as an optional module in LAMMPS since 2010 [16]. When used, the GPU package manages the host-device data transfers and launches the kernels for force computes and neighbor list builds on the device for individual MPI tasks. The neighbor list can also be built on the host and subsequently transferred to the device memory. This is relevant to the case where the simulated system is modeled by more than one pair styles each requiring a separate neighbor list. At the same time, the MPI process handles other force computes, including 
bonds, angles, dihedrals and impropers. Once the force computation on the device completes, the forces, energy and virial components are copied from the device memory back to the host memory. The GPU package offers three compile-time options for floating point precisions: single, double and mixed precision. In the mixed precision mode, atom positions are loaded as single precision numbers while forces, energy and virial components are tallied and stored as double-precision numbers in the device memory. Details on how the GPU package is implemented are given in Refs $[16,9,18]$.

Our implementation of the Tersoff potential is based on the previous work [18], where the three-body StillingerWeber (SW) potential was ported to the GPU package. Although we employ the same computing approach as in Ref. [18], the Tersoff potential is more computationally intensive than the SW potential, and hence requires special treatment as compared to the latter. We also modify the neighbor list indexing algorithm such that the three-body potentials supported by the GPU package can be combined with other potentials in a simulation, which was not available in previous work [18]. This is particularly relevant to systems modeled by a combination of force fields, e.g. carbon nanotubes-polymers and thin films-solid substrates.

\subsection{Tersoff potentials}

Considering a system of $\mathrm{N}$ atoms interacting via the Tersoff potential [2], the system potential energy cast in a pair-wise summation is given by:

$$
U=\frac{1}{2} \sum_{i=1}^{N} \sum_{j \neq i} V_{i j}
$$

where

$$
\begin{aligned}
V_{i j} & =f_{c}\left(r_{i j}\right)\left[f_{R}\left(r_{i j}\right)+b_{i j} f_{A}\left(r_{i j}\right)\right] \\
V_{i j}^{R} & =f_{c}\left(r_{i j}\right) f_{R}\left(r_{i j}\right) \\
V_{i j}^{A} & =f_{c}\left(r_{i j}\right) b_{i j} f_{A}\left(r_{i j}\right)
\end{aligned}
$$

and the cutoff function $f_{c}(r)$ is defined as:

$$
f_{c}(r)= \begin{cases}1, & r<R-D \\ \frac{1}{2}-\frac{1}{2} \sin \left(\frac{\pi}{2} \frac{r-R}{D}\right), & R-D<r<R+D \\ 0, & r>R+D,\end{cases}
$$

where $R$ are $D$ are model specific parameters. The two-body repulsion term $f_{R}(r)$ and attraction term $f_{A}(r)$ are given by:

$$
\begin{aligned}
& f_{R}(r)=A \exp \left(-\lambda_{1} r\right) \\
& f_{A}(r)=-B \exp \left(-\lambda_{2} r\right),
\end{aligned}
$$

where $A, B, \lambda_{1}$ and $\lambda_{2}$ are model specific parameters. The three-body interactions are given by $b_{i j}$ :

$$
\begin{aligned}
b_{i j} & =\left(1+\beta^{n} \zeta_{i j}^{n}\right)^{-\frac{1}{2 n}} \\
\zeta_{i j} & =\sum_{k \neq i, j} f_{C}\left(r_{i k}\right) g\left(\theta_{i j k}\right) \exp \left[\lambda_{3}^{m}\left(r_{i j}-r_{i k}\right)^{m}\right]
\end{aligned}
$$

where $\beta, \lambda_{3}, m$ and $n$ are model specific parameters. The angular dependent term $g(\theta)$ is given as:

$$
g(\theta)=\gamma_{i j k}\left(1+\frac{c^{2}}{d^{2}}-\frac{c^{2}}{d^{2}+\left(\cos \theta-\cos \theta_{0}\right)^{2}}\right)
$$


where $\theta$ is the angle between two vectors $\mathbf{r}_{i j}$ and $\mathbf{r}_{i k} \cdot \gamma_{i j k}, c, d$ and $\theta_{0}$ are model specific parameters. In LAMMPS the model specific parameters are read in from Tersoff files. The factor $b_{i j}$ captures the effects of the bonding order of the neighboring atoms of atom $i$ on atom $j$. It is important to note that $b_{i j} \neq b_{j i}$, even for single-element systems.

There are two variants of the Tersoff potential available in LAMMPS. In the first variant, another cutoff function is chosen [20]:

$$
f_{c}(r)=\left\{\begin{array}{l}
1, r<R-D \\
\frac{1}{2}-\frac{9}{16} \sin \left(\frac{\pi}{2} \frac{r-R}{D}\right)-\frac{1}{16} \sin \left(\frac{3 \pi}{2} \frac{r-R}{D}\right) \\
R-D<r<R+D \\
0, r>R+D
\end{array}\right.
$$

and the angular-dependent term is modified:

$$
\begin{aligned}
g(\theta) & =c_{1}+g_{0}(\theta) g_{a}(\theta) \\
g_{0}(\theta) & =\frac{c_{2}(h-\cos \theta)^{2}}{c_{3}+(h-\cos \theta)^{2}} \\
g_{a}(\theta) & =1+c_{4} \exp \left[-c_{5}(h-\cos \theta)^{2}\right]
\end{aligned}
$$

In the second variant, a close-separation pairwise modification based on a Coulomb potential and the Ziegler-BiersackLittmark (ZBL) universal screening function [21] is used in the energy function:

$$
\begin{aligned}
V_{i j} & =\left[1-f_{F}\left(r_{i j}\right)\right] V_{i j}^{\mathrm{ZBL}}+f_{F}\left(r_{i j}\right) V_{i j}^{\text {Tersoff }} \\
f_{F}\left(r_{i j}\right) & =\frac{1}{1+\exp \left[-A_{F}\left(r_{i j}-r_{c}\right)\right]} \\
V_{i j}^{\mathrm{ZBL}} & =\frac{1}{4 \pi \epsilon_{0}} \frac{Z_{1} Z_{2} e^{2}}{r_{i j}} \phi\left(r_{i j} / a\right) \\
a & =\frac{0.8854 a_{0}}{Z_{1}^{0.23}+Z_{2}^{0.23}} \\
\phi(x) & =0.1818 e^{-3.2 x}+0.5099 e^{-3.2 x}+0.2802 e^{-0.4029 x}+0.02817 e^{-0.2016 x}
\end{aligned}
$$

Despite their apparent differences, these variants of the Tersoff potential share the following common computation challenges: 1) the two-body repulsion terms, which can be handled in the same manner as for conventional pair-wise force fields and 2) the evaluation of the three-body terms in $\zeta_{i j}$ and $b_{i j}$, which are similar in the way the neighbor list of individual atoms is iterated. In the following sections, for compactness we will present only the implementation for the original form of the Tersoff potential. All the source code is given in the GPU package in the LAMMPS main repository available at http://lammps.sandia.gov/downloads.

We adopt the redundant computation approach (RCA) as proposed in Brown et al. [18], to ensure the output are deterministic in the sense that the results are identical for repeated runs with the same input and on the same number of MPI tasks. This is to separate the effects of unassociative floating-point arithmetic operations from possible bugs in the implementation for untested use cases. Furthermore, deterministic results are particularly important for massively parallel codes like LAMMPS when used for hardware-acceptance runs on supercomputers. In addition to having a deterministic algorithm for neighbor list builds [16], we avoid atomic operations in force compute kernels by performing the redundant computations for the three-body terms for each atom in the triplet. Listing 1 illustrates how the RCA is implemented for the three-body terms of the Tersoff potential.

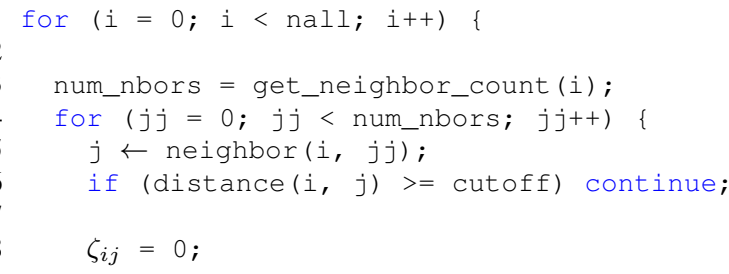




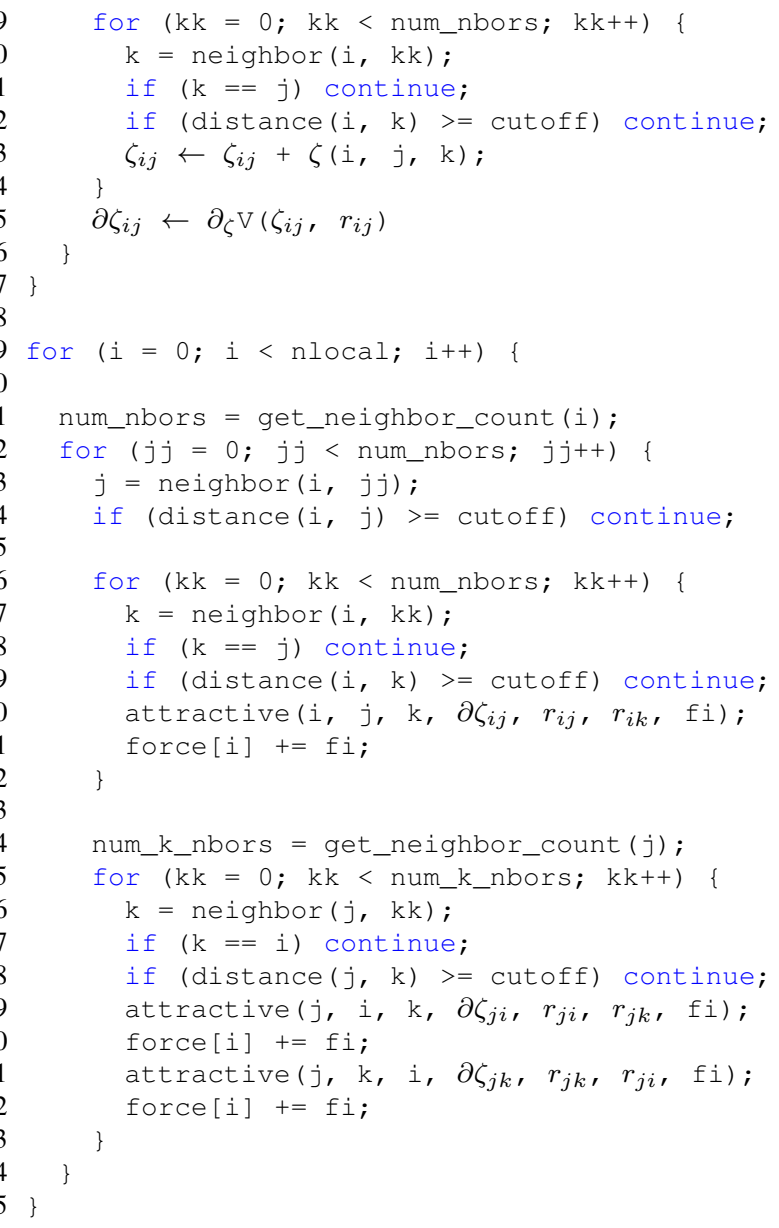

Listing 1: Redundant computation approach (RCA)

The first for loop (lines 1-17 in Listing 1) computes $\zeta(i, j)$ by going through the list of neighbors of $i$ for each neighbor $j$. The matrix $\zeta_{i j}$ has the size of nall by max_nbors, where nall is the total number of local and ghost atoms owned by the MPI process and max nbors is the maximum number of neighbors per atom. The matrix is allocated only on the device and resized only if nall or max_nbors grows after the neighbor list is rebuilt.

\section{a}

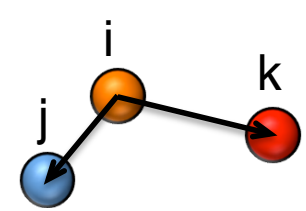

b

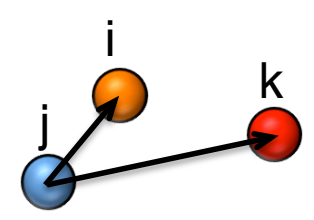

Figure 1: Given a triplet $(i j k)$, the forces on atom $i$ are computed when: (a) $j$ and $k$ are neighbors of $i: r_{i j}<r_{c}$ and $r_{i k}<r_{c}$; (b) $i$ and $k$ are neighbors of $j: r_{j i}<r_{c}$ and $r_{j k}<r_{c}$.

The second for loop (lines 19-45) computes the force on local atoms $i$. The first inner loop (lines 26-32) considers the case where both atoms $j$ and $k$ are the neighbors of atom $i$ (Fig. 1(a)). It loops through the neighbor list of $i$ and for each neighbor $j$ looks for the other neighbors $k(k \neq j)$ that are within the cutoff distance from $i$. Note that the force depends on the value of $\zeta(i, j)$ previously computed. 
The last inner loop (lines 35-43) computes the force on atom $i$ where $i$ is in the neighbor list of $j$, with $j$ being either local or ghost atoms (Fig. 1(b)). This time the loop goes through the neighbor list of the neighbor $j$ and looks for the neighbors $k(k \neq i)$ that are within the cutoff distance from $j$. For each $k$ the force on $i$ comes from both $\zeta(j, i)$ (line 39) and $\zeta(j, k)$ (line 41). It is therefore evident that if we did not precompute and store $\zeta(i, j)$ for reuse, the amount of calculations in the last inner loop would be increased by a factor of $b_{n}$, where $b_{n}$ is the average number of neighbors per atom.

It is important to point out that the RCA implementation described in Listing 1 is in fact not restricted to the Tersoff potentials, but applicable to any three-body potential in which there is no symmetry in the interaction between the bond order of the constituent atoms in any triplet, i.e. $b_{i j} \neq b_{j i}$.

\subsection{Implementation}

To port the RCA in the single-instruction multiple-thread parallelism on the GPUs, we implement three separate kernels, performing the work of the loops described in the previous section. The first kernel, called compute_zeta computes $\zeta(i, j)$, three-body prefactors, forces and energies, and stores the values for each pair $(i, j)$ on the device memory for reuse. The two remaining kernels, threebody_center and threebody_end, compute the force and energy for each local atom $i$ using the three-body prefactors just calculated. We employ the static regular force decomposition (SRFD) scheme as proposed in Ref. [18] where the force of atom $i$ is tallied from $w_{n}^{2}$ work-items (i.e. threads) with $w_{n}$ work-items performing the innermost loop for each neighbor, $j j$. The SRFD scheme allows for more efficient use of the device as to keep the GPU cores busy as much as possible for small atom counts per GPU. To avoid the synchronization barriers when tallying the forces and energies across the work-items for atom $i$, we restrict $w_{n}^{2}$ to be less than the size of a warp, i.e. 32 work-items in the current NVIDIA GPUs. For $w_{n}=1$, the SRFD scheme is equivalent to an atom decomposition scheme.

Listing 2 shows the pseudo-code of the kernel compute_zeta. The variable nbor_pitch corresponds to the total number of atoms of which the neighbor list is built, which is equal to nall $\times w_{n}$ if the neighbor list is built on the device. The variables of $f_{s e t} j$ and offset_k determine the starting indices for the outer and inner loops, respectively. In each thread, the inner loop accumulates $z$, a local variable. At the end of the inner loop, $\zeta(i, j)$ is computed by tallying the local variable $z$ across $w_{n}$ work-items via binary reduction, i.e. using the shf $1_{-}$xor () commands in CUDA Toolkit 7.0 and later for GPUs with compute capability 3.5 and higher. An OpenCL equivalence of this operation is also available in the GPU package in LAMMPS.

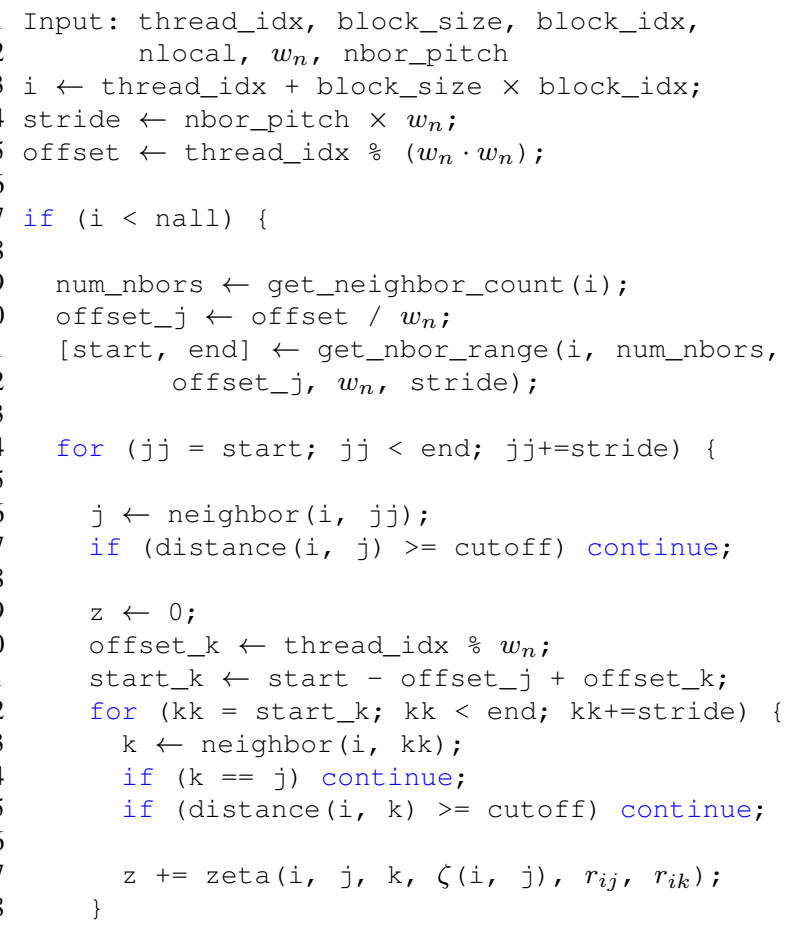


Listing 2: Pseudo-code for the kernel compute_zeta

The kernel threebody_center (Listing 3) looks similar to compute_zeta in the way the inner and outer loops iterate through the neighbor list of the atoms $i$. However, only the local atoms (i.e. $i<$ nlocal) need to be considered here. In each thread, the loops accumulate the forces (and energies) from the neighbors into a local variable fi. Finally, the local variable $\mathrm{f} i$ is accumulated into the force of $i$ by tallying across the $w_{n}^{2}$ work-items, also via binary reduction.

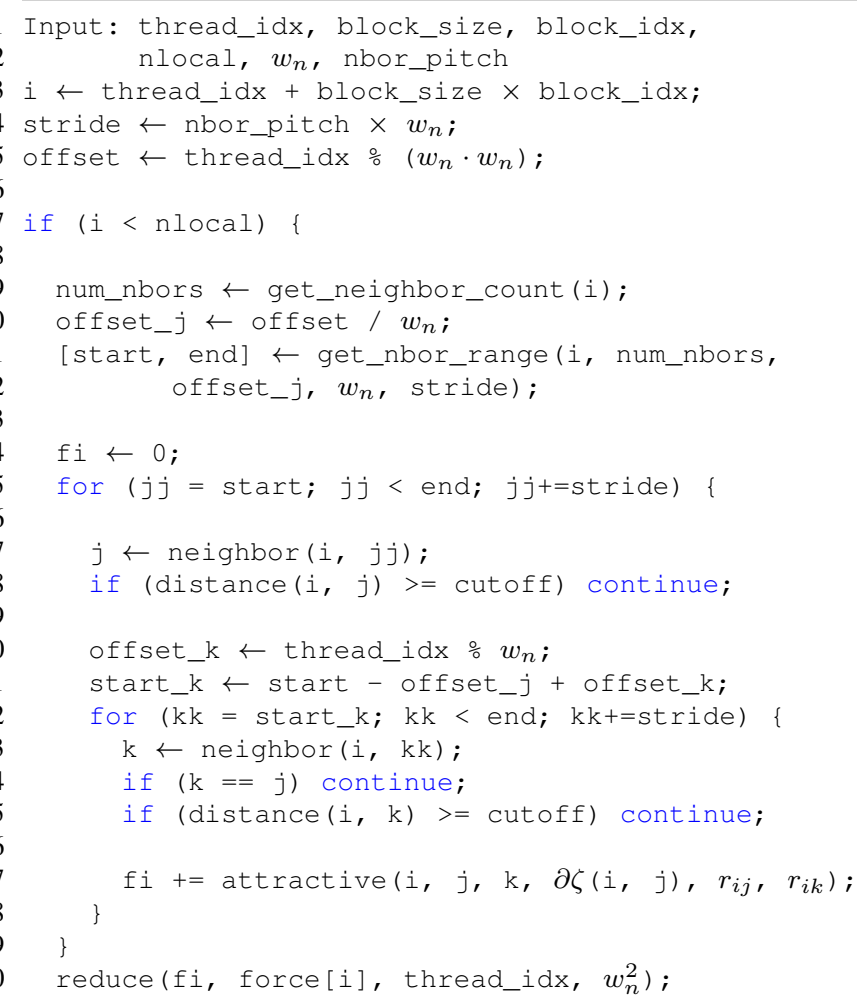

Listing 3: Pseudo-code for the kernel threebody_center

The kernel threebody_end (Listing 4) is similar to threebody_center in how the outer loop iterates the neighbor list of local atoms $i$. First, it loads $\zeta(j, i)$ to compute the force (and energy) that is distributed equally among $w_{n}$ work-items (line 20). Next, the index range in the neighbor matrix of the neighbors of atoms $j$ is determined. The inner loop then goes through the neighbor list of $j$ (lines 26-32), which can be either local or ghost atoms, in a similar manner to that in the other kernels, to compute and accumulate the force on the local atoms $i$.

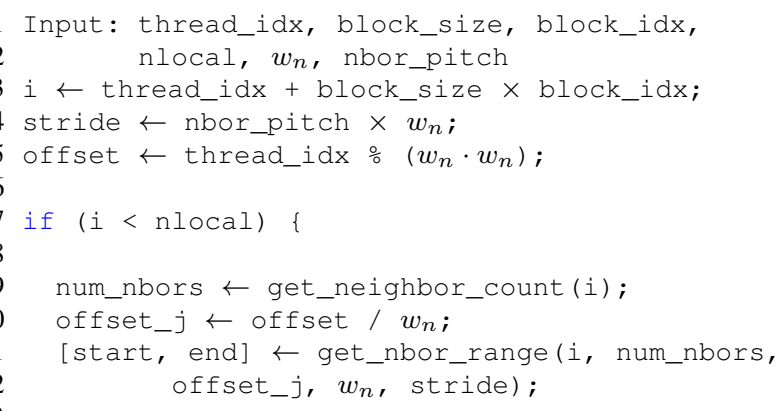




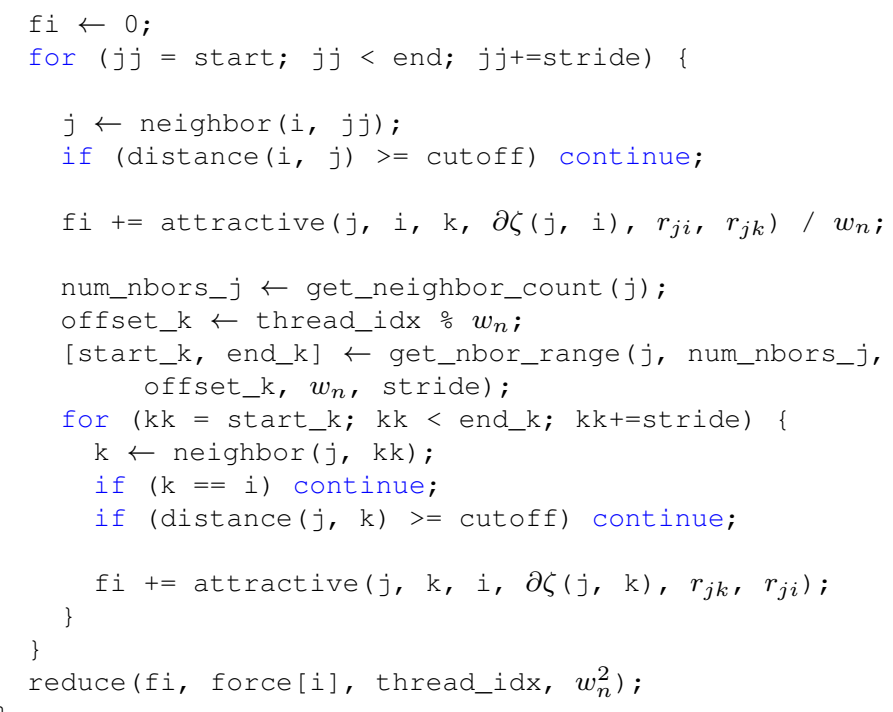

Listing 4: Pseudo-code for the kernel threebody_end

In order to handle multi-element systems, we extend the previous work [18] by adding arrays that map atom types to elements and elements to the corresponding parameter sets. The parameter sets are then stored as arrays on the device global memory once before the run. In each kernel, the parameter sets are then loaded into the shared memory where all the work items in a work group can get access.

It was assumed in the previous work [18] that all the atoms in the system interact via a single three-body force field, e.g. the Stillinger-Weber potential, which then allows direct access to the neighbors of neighbors of any given atom through the neighbor indices. In practice, however, there are many situations where the Tersoff potential is used to represent the interaction among a subset of the atoms in a system. The other non-bonded interactions are modeled by other force fields, such as ZBL, EAM or a combination of Lennard-Jones and Coulombic interactions. In such cases, although all the force fields in use may be supported by the GPU package, the neighbor lists associated with individual force fields must be built on the host before transferred to the device. We modify the neighbor list indexing scheme to accommodate these use cases by adding an extra array to map the items in the neighbor list to actual atom indices. This extra array is to be used in the kernel threebody_end, where the inner loop goes through the neighbor list of atoms $j$. These modifications do not affect the performance of the implementation for the previous use cases, i.e. all the atoms interacting via a single three-body force field.

\subsection{Benchmarks}

We select two types of systems typical of simulation studies in practice to characterize the performance of our implementation. The first system is a liquid box of 1:1 mixture of Ga: $\mathrm{N}$ equilibrated at $\mathrm{T}=1200 \mathrm{~K}$ (Fig. 2(a)); the other is a crystalline structure of Si equilibrated at $\mathrm{T}=100 \mathrm{~K}$ (Fig. 2(b)). The atoms are highly mobile in the liquid system while only fluctuating around their equilibrium positions in the crystalline structure. For the liquid system, we also vary the system density to examine the change in the performance of our implementation, as the computational load is expected to increase with the average number of neighbors per atom. All the benchmark runs take 1000 time steps so that the accelerator warmup time, if any, becomes negligible compared to the loop time. For compactness, we choose to only show the performance of the GPU version of the original Tersoff potential in the following sections as our preliminary runs indicated that the other variants would have similar results.

For the 1:1 mixture of Ga:N liquid system, the maximum cutoff distance given by the Tersoff parameters is $5.1 \AA$, the skin distance is chosen to be $2.0 \AA$ and the neighbor list is rebuilt every 5 time steps. Likewise, for the Si diamond cubic structure, the maximum cutoff distance is $4.2 \AA$, the skin distance is chosen to be $2.0 \AA$ and the neighbor list is rebuilt every 10 time steps. The time step interval for time integration is $1.0 \mathrm{fs}$. With these settings, there is no dangerous neighbor list build in all the runs. 
a

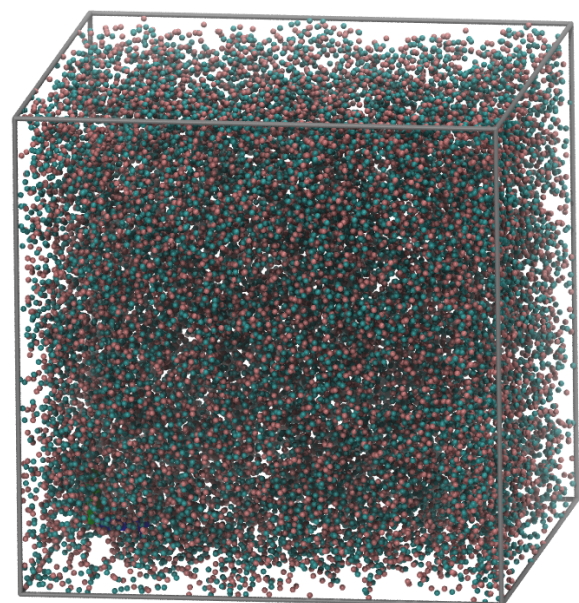

b

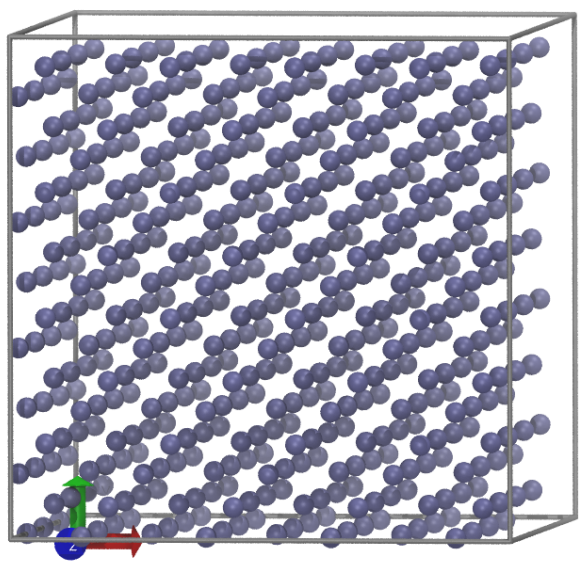

Figure 2: Example snapshots of two benchmark systems: (a) a 1:1 mixture of $\mathrm{Ga}: \mathrm{N}$ after relaxation at $\mathrm{T}=1200 \mathrm{~K}$; (b) a diamond cubic crystal structure of Si equilibrated at $\mathrm{T}=100 \mathrm{~K}$.

Benchmark runs were performed at two computing clusters: the Titan Cray XK7 supercomputer at the Oak Ridge National Laboratory, and the NVIDIA proprietary development (PSG) cluster. Depending on the hardware configuration of the computing clusters (details below), we use the C++ compilers, MPI libraries and CUDA toolkits available. LAMMPS is built with the April 2016 version. All the CPU-only runs were performed with the original version of the Tersoff potentials available in the MANYBODY package in LAMMPS.

\subsection{Validation}

We validate the correctness of our implementation using static and dynamic tests. In the static test, we compare the potential energy and atom forces obtained from the GPU-accelerated version with those from the CPU-only version for a given atom configuration. For this test, we equilibrate a 1:1 mixture of Ga: $\mathrm{N}$ with $\mathrm{N}=32000$ atoms in a cubic box of $160 \AA$ in size at $\mathrm{T}=1200 \mathrm{~K}$ to generate a set of 3000 configurations. For each configuration, the system potential energy and atom forces are computed with the CPU version and with the GPU-accelerated version in mixed precision and in double precision. The difference in potential energy between the GPU-accelerated and CPU-only runs is defined as

$$
\Delta U=\left|U_{\mathrm{GPU}}-U_{\mathrm{CPU}}\right|
$$

and the difference in atom forces as:

$$
\Delta f=\frac{1}{N} \sum_{i=1}^{N}\left\|\mathbf{f}_{i, \mathrm{GPU}}-\mathbf{f}_{i, \mathrm{CPU}}\right\| .
$$

In the double precision mode, the GPU-accelerated results are identical to those obtained from the CPU-only run, within the machine numerical precision. In the mixed precision mode, the difference between the GPU-accelerated run and the CPU run in potential energy is $\Delta U=(5.0 \pm 0.1) \times 10^{-6} \mathrm{eV}$ per atom and in atom forces $\Delta f=$ $(4.0 \pm 0.4) \times 10^{-9} \mathrm{eV} / \AA$ per atom, which are negligible relative to their corresponding absolute values.

In the dynamic test, we compare GPU and CPU runs with regards to thermodynamic quantities such as system potential energy, temperature and pressure over equilibration runs from the same initial configurations. In Fig. 3 we show these quantities during the equilibration of the Ga: $\mathrm{N}$ mixture consisting of $\mathrm{N}=32000$ atoms at $\mathrm{T}=1200 \mathrm{~K}$. It appears that the GPU and CPU-only runs give statistically indistinguishable results. A similar observation is found with the equilibration of a crystalline structure consisting of $\mathrm{N}=32768$ silicon atoms in the canonical ensemble at $\mathrm{T}$ $=100 \mathrm{~K}$. 

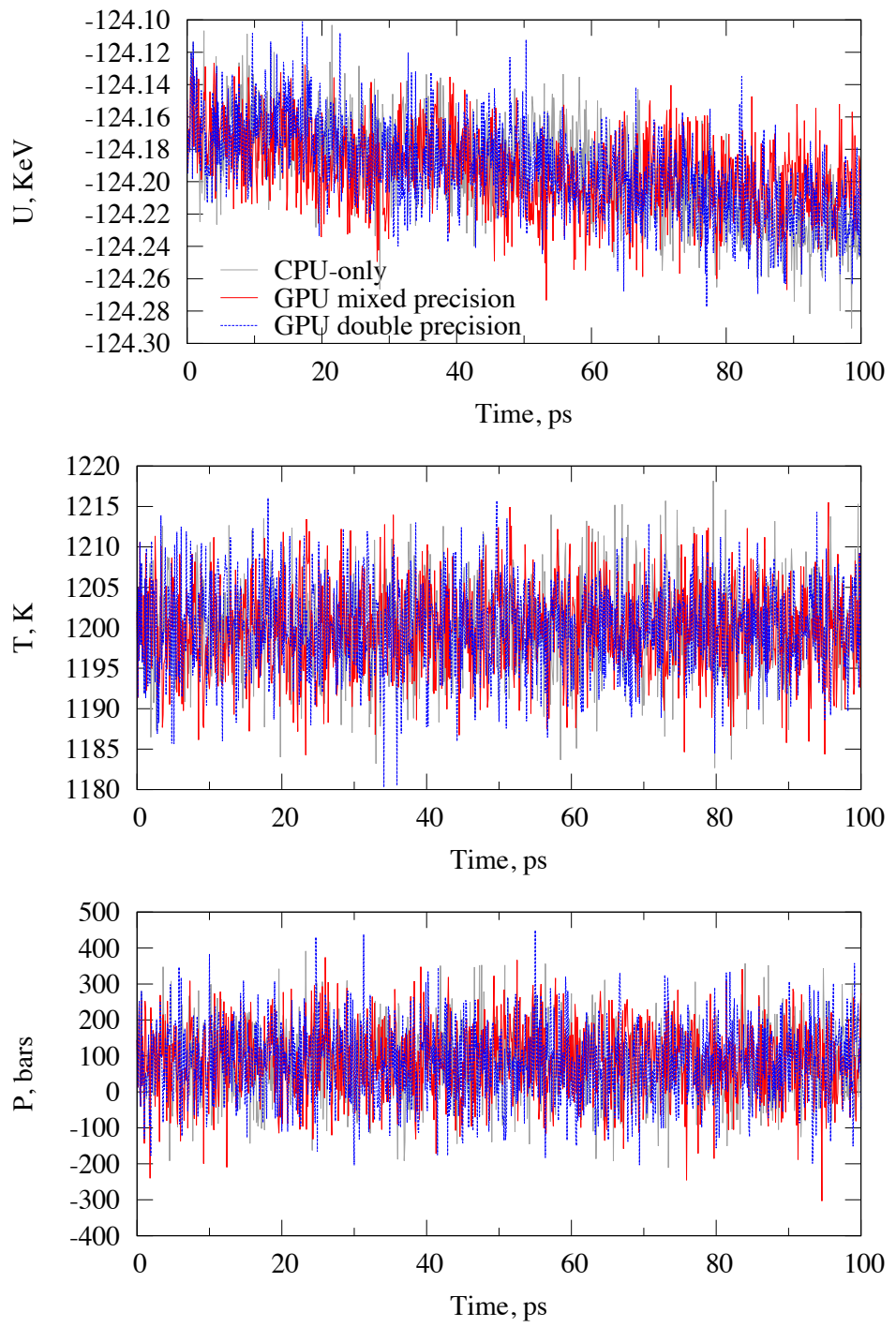

Figure 3: Comparison between GPU-accelerated and CPU-only equilibration runs for a 1:1 mixture of Ga:N with $\mathrm{N}=32000$ atoms, in terms of several thermodynamic quantities: (a) system potential energy, (b) temperature and (c) pressure. The CPU run was performed with 16 MPI tasks. The GPU-accelerated runs used 2 MPI tasks each with a GK210 GPU on a Tesla K80 card.

\section{Results}

\subsection{Single node performance}

We first investigate the performance of our implementation on a single node where the interprocess communication overhead is not affected by network latency. The single-node performance is particularly relevant for simulation studies conducted on small-scale workstations where off-node communication overhead is usually prohibitive. The benchmark is performed on the NVIDIA PSG cluster where each nodes has dual 16-core Intel E5-2698 v3 CPUs running at $2.3 \mathrm{GHz}$ and 4 Tesla K80 GPU cards. LAMMPS is built with the Intel C++ 15.0 compiler and Intel MPI 10.1.2 library with the "-O2" optimization flag. The GPU package is built with mixed precision using the NVIDA CUDA 7.5 toolkit with "-O3 -use-fast-math" optimization flags.

Fig. 4 shows the strong scaling behavior of the GPU-accelerated version for multiple GPUs compared to 16-MPI CPU-only runs. (It makes little sense to compare with single CPU runs as LAMMPS is designed for MPI parallelism.) In this analysis, the 1:1 mixture of Ga: $N$ consists of $\mathrm{N}=256000$ atoms in a cubic box of $200 \AA$ in size and was relaxed 
at $1200 \mathrm{~K}$ for $50 \mathrm{ps}$ to generate initial configurations for the benchmark runs. Likewise, the Si diamond cubic structure consists of $\mathrm{N}=262144$ atoms in a cubic box of $173.8 \AA$ in size, and was equilibrated for 50 ps.

In multiple GPU runs, we report the timings for 1 MPI task per GPU, which is the optimal number of MPI tasks per GPU for the studied systems. Note that each Tesla K80 card contains two GK210 GPUs, and that the GPU package detects the number of GPUs available in each node and assigns MPI tasks to each GPU. As the Tesla K80 cards are used with autoboost enabled, the core clock rate can be as high as $0.82 \mathrm{GHz}$ depending on the available thermal and power budget on the nodes.

For the Ga:N system (Fig. 4(a)), the performance of the GPU-accelerated version on a Tesla K80 card is 2.1 ns/day, 2.2X faster than that of the 16-MPI task CPU-only run (0.95 ns/day). For 4 Tesla K80 cards (8 GK210 GPUs), the GPU accelerated version yields a speedup of 7.9X and a parallel efficiency of $88 \%$ considering the single GPU timing as the baseline. As can be seen, the GPU implementation exhibits a good strong scaling behavior as the atom count per GPU is reduced to 32000 . Note that the simulated system chosen is in liquid state, where each atom has 22 neighbors on average, including both non-interacting and interacting atoms. In this case, the optimal number of work-items $w_{n}$ is found to be 4 .

For the Si crystalline structure, we observe a similar strong scaling behavior (Fig. 4(b)). The GPU version yields $3.7 \mathrm{~ns} /$ day on a Tesla K80 card, 2.4X faster than the 16-MPI task CPU-only run. This performance value (3.7 ns/day) is on par with the performance reported in a recent work by Höhnherbach and coworkers [15] on a similar Si system. The performance gains on a single Tesla K80 cards and on 4 Tesla K80 cards versus the 16-MPI CPU-only run are slightly better than in the Ga:N system. The parallel efficiency is maintained at $85 \%$ for 4 Tesla K80 cards. For this crystalline system, where the average number of neighbors per atom is 16 , we found that the optimal number of work-items $w_{n}=2$. The influences of $w_{n}$ on the performance will be discussed later.

(a)

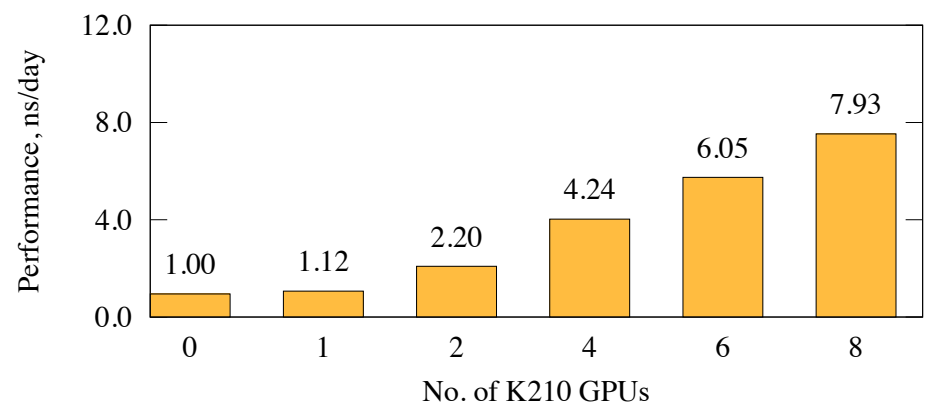

(b)

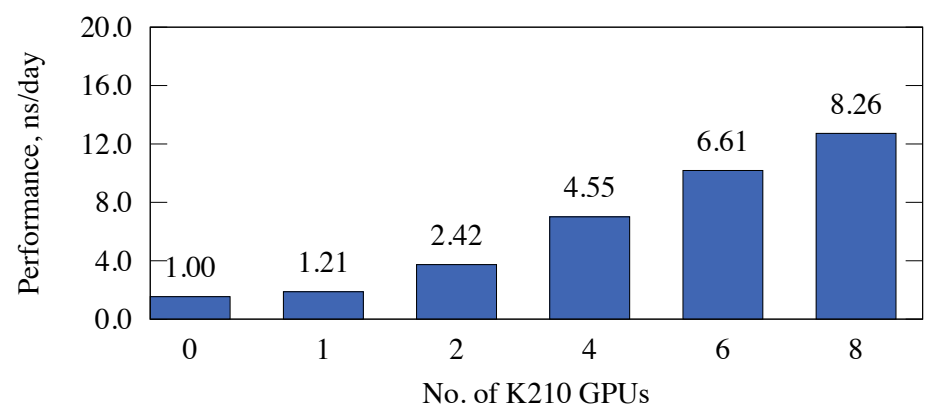

Figure 4: Strong scaling results for (a) a 1:1 mixture of Ga:N containing N $=256000$ atoms already equilibrated in the liquid state at $\mathrm{T}=1200 \mathrm{~K}$ and (b) a diamond cubic structure of Si containing $\mathrm{N}=266144$ atoms, with Tesla K80 cards on the NVIDIA PSG cluster. The number on top of each bar is the speedup compared to the run with 16 MPI tasks without GPU acceleration (i.e. number of GPUs is zero). The reported performances are measured for 1000-step runs. For each data point, 10 runs are performed and the averaged performances are reported.

Because the GPU package can be compiled with OpenCL, it may be useful to compare the performances of OpenCL and CUDA builds. We compile the kernels with the optimization flags "-cl-fast-relaxed-math -cl-mad- 
enable" using the OpenCL library included in the CUDA 7.5 Toolkit. The optimal execution configuration for the OpenCL runs, e.g. block sizes for force computes and neighbor builds, are manually tuned for Kepler GPUs. As shown in Table 1, the performance of the OpenCL build is slower than that of the CUDA build by approximately 5\% on the given NVIDIA GPUs. The strong scaling behavior of the OpenCL build is similar to that of the CUDA build up to 8 GK210 GPUs, i.e. with 32000 atoms per GPU. The difference in performance is not significant, despite the fact that OpenCL targets generic devices. Nevertheless, we did not have opportunities to test our implementation on other GPU vendors to have a more thorough comparison.

\begin{tabular}{ccc}
\hline No. of K210 GPUs & OpenCL, ns/day & CUDA, ns/day \\
\hline 1 & 1.03 & 1.07 \\
2 & 1.97 & 2.09 \\
4 & 3.82 & 4.03 \\
6 & 5.46 & 5.74 \\
8 & 7.18 & 7.53 \\
\hline
\end{tabular}

Table 1: Comparison in the performance of OpenCL and CUDA builds on Tesla K80 cards. Performances are measured for 1000 MD-step runs. The benchmark system is the same $\mathrm{N}=256000$ atom system as earlier.

Finally, we observe that the GPU-accelerated runs in the double precision mode are about 2.0X slower than in the mixed precision mode for all the cases we examined. This is consistent with most force fields supported by the GPU package.

\subsection{Multiple node performance}

For massively parallel codes like LAMMPS, it is important to maintain a considerable parallel efficiency at high node counts. We examine both strong and weak scaling behaviors of the GPU-accelerated version across multiple compute nodes. For this purpose, we simulate the same Ga:N system as above on the OLCF Titan supercomputer. Each Titan compute node has a 16-core AMD Opteron $6274 \mathrm{CPU}$ running at $2.2 \mathrm{GHz}$ and a K20X GPU. In all the runs, with and without GPU acceleration, the MPI tasks are mapped with the non-uniform memory access (NUMA) nodes to minimize off-node communication.

The strong scaling behavior of the GPU-accelerated version for different system sizes (at the same density and temperature) is shown in Fig. 5(a). A linear strong scaling behavior means that the performance should increase linearly with the number of compute nodes with a fixed-size problem. We observe that for all the system sizes studied the performance deviates from linear scaling as the number of GPUs increases, which is to be expected as the interprocess communication overhead becomes significant relative to the computation time. As the system size increases, the performance decreases proportionally; however, the scaling behavior remains closer to linear scaling at higher atom counts per GPU. This is because the increase in the computation workload per GPU is greater than that in the interprocess communication overhead for higher atom counts. Table 2 summarizes the performances of the GPU-accelerated runs for the system sizes under investigation on a single node and on 64 nodes.

\begin{tabular}{rcc}
\hline System size & 1-node, ns/day & 64-node, ns/day \\
\hline 32000 & 4.27 & 72.2 \\
108000 & 0.94 & 31.6 \\
256000 & 0.53 & 23.0 \\
500000 & 0.35 & 18.2 \\
\hline
\end{tabular}

Table 2: Performance with GPU acceleration as function of the system size. The benchmark system is the $1: 1 \mathrm{mixture}$ of Ga: $\mathrm{N}$ at $\mathrm{T}=1200 \mathrm{~K}$. Speedup is the performance gain compared to the CPU-only runs with 16 MPI tasks per node. The number of work-items per atom is fixed at $w_{n}=4$.

Fig. 5(b) shows the strong scaling behavior of the GPU-accelerated runs and the CPU-only runs with 16 MPI tasks per node, up to 128 nodes for $\mathrm{N}=256000$ atoms. On a single node, the GPU-accelerated version yields a performance of $0.53 \mathrm{~ns} /$ day, while the 16-MPI task CPU-only runs get $0.39 \mathrm{~ns} /$ day. On 128 nodes, the GPU-accelerated runs are 2.2X faster than the CPU-only runs. It is also noteworthy that the parallel efficiency of the GPU-accelerated version 
is maintained at about $63 \%$ up to 64 nodes, and drops to $52 \%$ for 128 nodes as there are on average 2000 atoms per GPU. Compared to pairwise potentials with the same atom count per GPU, this parallel efficiency value is remarkably high, confirming that the Tersoff potential is computation intensive.

(a)

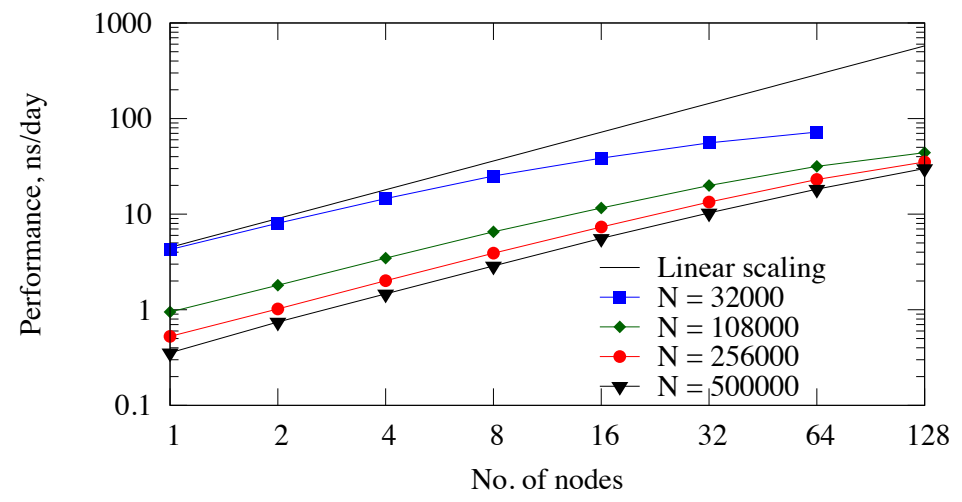

(b)

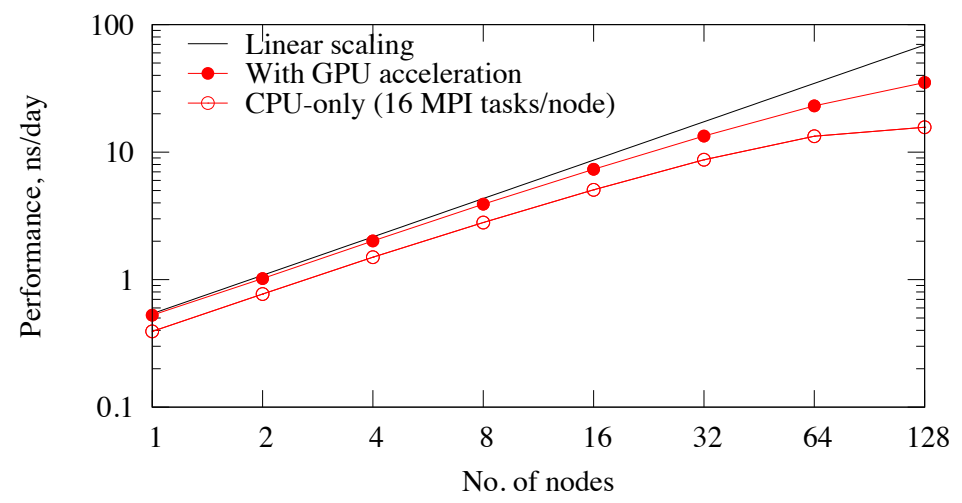

(c)

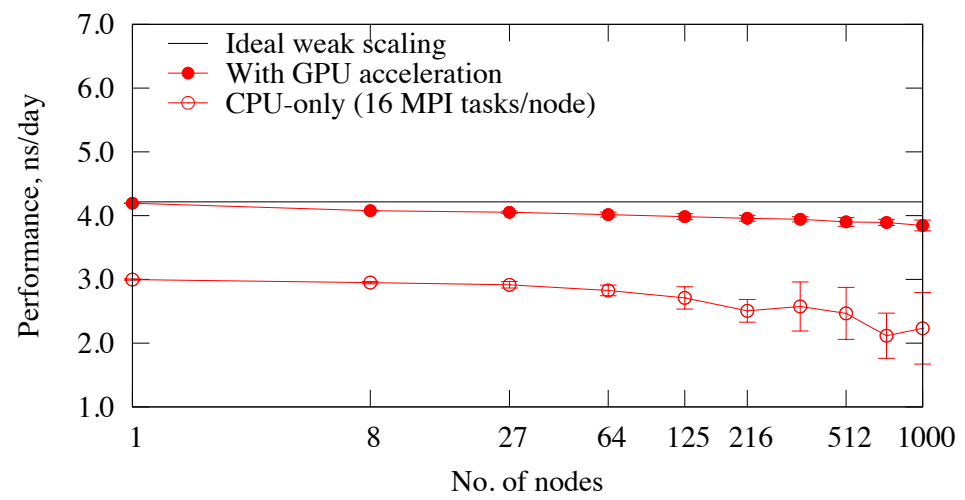

Figure 5: Performance across multiple compute nodes on the OLCF Titan supercomputer: (a) Strong scaling results for the 1:1 mixture of Ga:N equilibrated in the liquid state at $\mathrm{T}=1200 \mathrm{~K}$ with different numbers of atoms. (b) Strong scaling results for the $\mathrm{N}=256000$ atom system with GPU acceleration and with the MPI-flat version using 16-MPI tasks per node. (c) Weak scaling results with 32000 atoms per node for systems at the same liquid state. The reported performances are measured for $1000 \mathrm{MD}$ steps. The error bars are the standard deviations for 10 data sets.

The weak scaling behavior of the GPU-accelerated and CPU-only versions on Titan is shown in Fig. 5(c). An ideal weak scaling behavior means that the performance should remain constant with respect to the number of compute 
nodes with the number of atoms per node unchanged. In this analysis, we choose the number of atoms per node to be 32000 to ensure efficient utilization of the GPU on each node [16, 17]. The equilibrated unit cell is replicated by the same amount along the $\mathrm{x}, \mathrm{y}$ and $\mathrm{z}$ directions and the number of nodes to be used is set accordingly. For instance, the unit cell is replicated 2 times along $\mathrm{x}, 2$ times along y and 2 times along $\mathrm{z}$ directions when run on 8 nodes. As can be seen from Fig. 5(c), the parallel efficiency of the CPU-only runs decays faster than the GPU runs because the former require 16 times more MPI tasks than the latter, leading to more interprocess communication overhead. At 1000 nodes, i.e. with 32 million atoms, the GPU-accelerated runs achieved $4.0 \mathrm{~ns} / \mathrm{day}, 2.2 \mathrm{X}$ times faster than the CPU-only runs with 16000 MPI tasks, while maintaining a parallel efficiency of $93 \%$, considerably higher than that of the CPU-only version $(75 \%)$.

\begin{tabular}{ccc}
\hline No. of neighbors/atom & Performance, ns/day & Speedup \\
\hline 34 & 17.8 & 1.2 \\
22 & 22.9 & 1.7 \\
18 & 27.5 & 2.1 \\
7 & 52.2 & 1.5 \\
\hline
\end{tabular}

Table 3: Performance with GPU acceleration as function of the average number of neighbors per atom. The benchmark system is the 1:1 mixture of Ga: $\mathrm{N}$ containing $\mathrm{N}=256000$ atoms at different number densities. All the performance values are for 64 nodes. Speedup is the performance gain compared to the CPU-only runs with 16 MPI tasks per node. The number of work-items per atom is fixed at $w_{n}=4$.

We also vary the system number density by changing the box volume while keeping the number of atoms the same $(\mathrm{N}=256000)$. Table 3 shows that the performance and speedup of the GPU-accelerated version depends on the average number of neighbors per atom. As the average number of neighbors per atom decreases, the RCA generally helps decreases the workload per thread by a factor of $w_{n}^{2}$ at the expense of binary reduction for any given atom $i$. However, when the number of neighbors per atom is too small, i.e. at very low densities, the speedup of the GPU version drops, as the computational saving is considerably reduced relative to the overhead due to host-device data transfers and kernel launches, as expected for other force fields supported by the GPU package.

There are two key parameters that determine the performance of the GPU-accelerated Tersoff version: the number of work-items per atom, $w_{n}$, and the number of MPI tasks sharing a GPU. As pointed out by Ref. [18], the optimal value of $w_{n}$ depends on the average number of neighbors per atom because it determines the workload of the inner and outer loops per thread. Fig. 6 shows the effects of the number of work-items per atom, $w_{n}$, on the simulation time on a single compute node for the two systems under investigation.

For the Ga:N mixture (Fig. 6(a)), the optimal value is $w_{n}=4$, which gives a speedup of about $2.2 \mathrm{X}$ on a single Tesla K80 card compared to a 16-MPI task CPU-only run. Using $w_{n}=1$ is the least efficient, which is $1.7 \mathrm{X}$ faster than the 16-MPI task CPU-only run, suggesting that atom decomposition is not optimal for this particular system, where there are 22 neighbors per atom on average.

For the Si crystalline structure (Fig. 6(b)), where the average number of neighbors per atom is 16, the optimal value is $w_{n}=1$, which gives a speedup of $2.5 \mathrm{X}$ on a single Tesla K80 card compared to a 16-MPI task CPU-only run. Whereas, using $w_{n}=4$ is only $2.2 \mathrm{X}$ faster than the CPU-only run due to the increased overhead associated with tallying the forces across $w_{n}^{2}$ threads. It is also noteworthy that using GPU acceleration reduces the force compute contribution from $97 \%$ to about $83 \%$ with $w_{n}=1$. As a rule of thumb, the more the neighbors per atom, the higher the optimal value of $w_{n}$.

We did not see any substantial improvement when using multiple MPI tasks sharing the GPU in all the studied cases. The performance gain, if any, of using 2 or 4 MPI tasks per GPU compared to 1 MPI per GPU is within 10\%. As shown in Fig. 6, time integration and other tasks contribute to about $10-15 \%$ of the simulation time. The benefit from spatial decomposition with multiple MPI tasks for high atom counts per GPU by cutting the cost of time integration and force compute, is therefore shadowed by the overhead associated with oversubscribing the GPU, even in the presence of the NVIDIA multi-process server.

To further characterize the computational pattern of the GPU version, we employ the profiling tools included in the CUDA Toolkit. The three-body kernels indeed require a remarkably high amount of arithmetic operations associated with the calculation of the gradient of $f_{c}\left(r_{i j}\right)$ and $b_{i j}$. Particularly, the kernels become dominant for large atom counts per GPU, whereas the kernel compute_zeta is significant for small atom counts per GPU. This high arithmetic intensity and the inevitable branch divergences when looping through the neighbor lists are the key factors that prevent further 


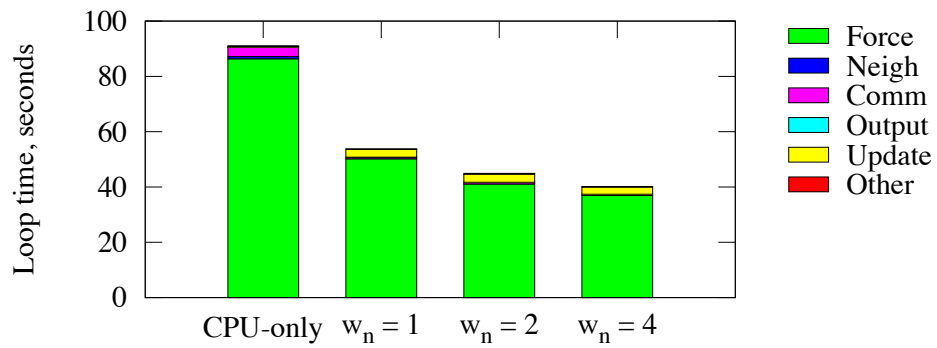

(b)

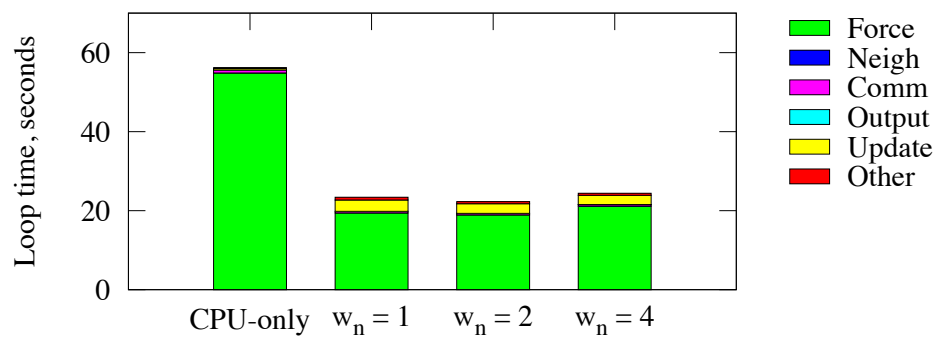

Figure 6: Effects of the number of work-items per atom in the inner loop on the loop time for (a) the Ga:N mixture, $\mathrm{N}=256000$ atoms, and (b) the Si crystalline system, $\mathrm{N}=262144$ atoms. The CPU-only runs are performed with 16 MPI tasks while the GPU runs with 2 MPI tasks each using $1 \mathrm{GK} 210 \mathrm{GPU}$ on a Tesla K80 card. The reported times are for $1000 \mathrm{MD}$ steps. Force $=$ force computation, Neigh $=$ time spent for neighbor list builds, Comm $=$ time spent for communication between MPI tasks, Output $=$ time spent for output, Update $=$ time spent for time integration in these runs, Other $=$ time for the remaining tasks (e.g. memory allocation/deallocation, data initialization, etc.)

performance improvement of our GPU implementation. Interestingly, we note that a single GK210 GPU on the Tesla K80 cards offers a speedup of about $2 \mathrm{X}$ compared to the GK110 GPU on the K20X cards despite the fact that the former has a smaller number of compute units (13 vs. 14) and a similar clock rate (with autoboost enabled). This is because the GK210 GPUs have a larger register file than the GK110 GPUs (512 KB vs. $256 \mathrm{~KB}$ ), thus allowing for more workgroups (i.e. thread blocks) to be executed concurrently.

\section{Discussion}

One of the main reasons the redundant computation approach (RCA) is pursued in the present study is to reduce the number of uncoalesced global memory writes as the memory bandwidth is often the bottleneck of most GPU applications. The RCA also helps ensure deterministic results, which are important for certain practical reasons. Additionally, with the RCA the interprocess communication for force accumulation from ghost atoms is removed. Nevertheless, as pointed out by previous work [18], the tradeoffs of using the RCA include the increased number of calculations (up to $3 \mathrm{X}$ compared to algorithms that involve atomic operations), extra calculation of neighbor lists for ghost atoms, and an increase in the number of ghost atoms with doubling the size of the border region. Among these, the increased number of calculations in three three-body kernels leads directly to the high arithmetic intensity described in the previous section. As a consequence, the speedup of the GPU-accelerated Tersoff potential versus the flat MPI version is noticeably reduced for large numbers of neighbors per atom. At the other extreme, i.e. when each atom has very few neighbors, the performance boost from the GPU package in general, and the RCA in particular, diminishes as the computation time saved is smaller than the time spent for host-device data transfers and kernel launches.

It is important to note that the current implementation requires storing the three-body prefactors for owned and ghost atoms on the device memory, which we consider a shortcoming of the RCA when applied to the Tersoff potentials. While storing this matrix reduces redundant computations, it leaves a large memory footprint per MPI task on the device, limiting the upper bound of the atom count per GPU. For instance, we found that the maximum number 
of atoms per MPI task that can be accommodated on a single Tesla K20X GPU is about 600000 for the liquid Ga:N system under investigation.

We stress that the RCA implementation is applicable to a family of three-body potentials where there is no symmetry in the forces on individual atoms in a triplet. In other words, $(i j k),(i k j),(j k i),(j i k),(k i j)$ and $(k j i)$ should be treated as different triplets when we compute the force on atom $i$. In this regard, the Stillinger-Weber potential is a special case of this family, where the number of distinct triplets is effectively reduced by half. As we have already showed, three variants of the Tersoff model can be implemented using the same approach, the only changes needed are in the formulae for specific analytical terms. We envision that our implementation can be extended to other three-body force fields sharing such a common feature, such as the charge-optimized many-body (COMB) potentials [22].

Because our aim is to have an implementation independent of the nature of the systems (e.g. liquid or solid phases, multiple-component systems and combined force fields), we did not make any assumption on the neighbor list of individual atoms, nor attempt any further optimization, e.g. to filter out non-interacting atoms, i.e. those are outside the cutoff distance, in the neighbor list at each time step. If the number of interacting atoms in the neighbor list is small, or if deterministic results are not important, alternative schemes could be more efficient, which involve atomic operations to avoid redundant calculations without much compromise in accuracy (e.g. see Refs. [13] and [15]). In such cases, although our implementation may not offer the best possible performance, its compatibility with non-accelerated functionalities in LAMMPS (e.g. specialized boundary conditions, application of constraint forces, simulation box deformations, calculation of diagnostic properties) makes it competitive to the other alternatives for practical simulations. As such, our implementation benefits LAMMPS users with another viable option, in addition to using the Kokkos and USER-INTEL packages, for running large-scale simulations with Tersoff potentials efficiently on hybrid computing platforms. Finally, it recently comes to our attention that the performance of the CPU-only version of the three-body potentials in LAMMPS can be substantially improved by using shorter temporary neighbor lists for individual atoms in the evaluation of the three-body terms. We will investigate this promising optimization strategy in the coming release of the GPU-accelerated version of the Stillinger-Weber and Tersoff potentials.

\section{Conclusion}

We have implemented the GPU-accelerated version of several variants of the Tersoff potential for LAMMPS with an eye toward a broad range of modeling applications, including multiple-element systems, liquid and solid phases and combined force fields. The GPU-accelerated version is faster than the CPU-only version on 16 MPI tasks per node by a factor of 2.0-2.5X depending on the average number of atoms per GPU, the average number of neighbors per atom and the GPU specifications. Importantly, our implementation exhibits good scalability up to 1000 compute nodes and its performance is primarily bound by the accelerator capacity. Compared to other GPU implementations of the Tersoff potentials to date, ours takes full advantage of the MPI parallelization and other functionalities in LAMMPS, making it ready for production runs both on small-sized workstations and on hybrid MPI/accelerator supercomputers.

\section{Acknowledgments}

T. D. N. thanks W. M. Brown for the help with the GPU package framework and for the discussion on the implementation of three-body potentials and A. Kohlmeyer for helpful discussion when debugging the cases where the Tersoff potentials are combined with other force fields in a simulation. We gratefully acknowledge the support from the NVIDIA Corporation for access to the Tesla K80 GPUs for this study. This research used resources of the Oak Ridge Leadership Computing Facility at Oak Ridge National Laboratory, which is supported by the Office of Science of the Department of Energy under Contract DE-AC05-00OR22725. This research is funded by Vietnam National Foundation for Science and Technology Development (NAFOSTED) under grant number 103.01-2015.52.

\section{References}

1. F. H. Stillinger, T. A. Weber. Computer simulation of local order in condensed phases of silicon. Phys. Rev. B, 31:5262, 1985.

2. J. Tersoff. New empirical approach for the structure and energy of covalent systems. Phys. Rev. B, 37:6991, 1988.

3. S. J. Plimpton, A. P. Thompson. Computational aspects of many-body potentials. Mat. Res. Soc. Bulletin, 37:513-521, 2012. 
4. J. C. Phillips, R. Braun, W. Wang, J. Gumbart, E. Tajkhorshid, E. Villa, C. Chipot, R. D. Skeel, L. Kale, K. Schulten. Scalable molecular dynamics with NAMD. Mat. Res. Soc. Bulletin, 26:1781-1802, 2005.

5. H. J. C. Berendsen, D. van der Spoel, R. van Drunen. GROMACS: A message-passing parallel molecular dynamics implementation. Comput. Phys. Commun., 91:43-56, 1995.

6. S. J. Plimpton. Fast parallel algorithms for short-range molecular dynamics. J. Comp. Phys., 117:1-19, 1995.

7. J. A. Anderson, C. D. Lorenz, A. Travesset. General purpose molecular dynamics simulations fully implemented on graphics processing units. J. Comp. Phys., 227:5342-5359, 2008.

8. I. V. Morozov, A. M. Kazennova, R. G. Bystryia, G. E. Normana, V. V. Pisareva, V. V. Stegailova. Molecular dynamics simulations of the relaxation processes in the condensed matter on GPUs. Comput. Phys. Commun., 182:1974-1978, 2011.

9. W. M. Brown, T. D. Nguyen, M. Fuentes-Cabrera, J. D. Fowlkes, P. D. Rack and M. Berger, A. S. Bland. An evaluation of Molecular Dynamics performance on the hybrid Cray XK6 supercomputer. Procedia Comput. Sci., 9:186-195, 2012.

10. C. Hou, W. Ge. GPU-accelerated molecular dynamics simulation of solid covalent crystals. Mol. Sim., 38:8-15, 2012.

11. C. Hou, J. Xu, P. Wang, W. Huang, W. Ge. Efficient GPU-accelerated molecular dynamics simulation of solid covalent crystals. Comput. Phys. Commun., 184:1364-1371, 2013.

12. C. Hou, M. Li, Y. Zhou, J. Cui, Z. Cui, J. Wang. Molecular dynamics simulations with many-body potentials on multiple GPUs-The implementation, package and performance. Comput. Phys. Commun., 184:2091-2101, 2013.

13. A. S. Minkin, A. B. Teslyuk, A. A. Knizhnik, B. V. Potapkin. GPGPU performance evaluation of some basic Molecular Dynamics algorithms. IEEE High Performance Computing and Simulation (HPCS), 2015 International Conference on, 629-634, 2015.

14. W. M. Brown, J.-M. Carillo, N. Gavhane, F. M. Thakkar, S. J. Plimpton. Optimizing legacy molecular dynamics software with directive-based offload. Comput. Phys. Commun., 195:95-101, 2015.

15. M. Hohnerbach, A. E. Ismail, P. Bientinesi. The vectorization of the Tersoff multi-body potential: An exercise in performance portability. arXiv:1607.02904v1 [cs.CE], 2016.

16. W. M. Brown, P. Wang, S. J. Plimpton, A. N. Tharrington. Implementing Molecular Dynamics on Hybrid High Performance Computers Short range Forces. Comput. Phys. Commun., 182:898-911, 2011.

17. W. M. Brown, A. Kohlmeyer, S. J. Plimpton, A. N. Tharrington. Implementing Molecular Dynamics on Hybrid High Performance Computers - Particle-Particle Particle-Mesh. Comput. Phys. Commun., 183:449-459, 2012.

18. W. M. Brown, Y. Masako. Implementing Molecular Dynamics on Hybrid High Performance Computers - Three-body potentials. Comput. Phys. Commun., 184:2785-2793, 2013.

19. T. D. Nguyen, S. J. Plimpton. Accelerating dissipative particle dynamics simulations for soft matter systems. Comput. Mater. Sci., 100:173180, 2015.

20. T. Kumagai, S. Izumi, S. Hara, S. Sakai. Development of bond-order potentials that can reproduce the elastic constants and melting point of silicon for classical molecular dynamics simulation. Comput. Mater. Sci., 39:457-464, 2007.

21. J. F. Ziegler, J. P. Biersack, U. Littmark. Stopping and ranges of ions in Matter. Pergamon Press, 1:457, 1985.

22. T.-R. Shan, B. D. Devine, T. W. Kemper, S. B. Sinnott, S. R. Phillpot. Charge-optimized many-body potential for the hafnium/hafnium oxide system. Phys. Rev. B, 81:125328, 2010. 